\title{
An Effective Subdivision Algorithm for Diffuse Scattering of Ray Tracing
}

\author{
Mingming Gan ${ }^{1}$, Xuhong Li $^{2}$, Fredrik Tufvesson ${ }^{2}$, Thomas Zemen ${ }^{1}$ \\ ${ }^{1}$ Forschungszentrum Telekommunikation Wien (ftw.), Vienna, Austria \\ ${ }^{2}$ Department of Electrical and Information Technology, Lund University, Lund, Sweden \\ Contact: gan@ftw.at
}

\begin{abstract}
Accurate modeling of electromagnetic wave propagation by means of ray tracing (RT) includes not only specular reflection, penetration through dielectric blocks and diffraction, but also diffuse scattering mechanisms. The accuracy, supported by a precise description of the environment, is achieved at a very high computational complexity. This computational complexity scales directly with the number of propagation paths, of which the diffuse scattering paths comprise a large proportion. In this paper, we propose a general subdivision algorithm for diffuse scattering of RT in indoor scenarios based on concentric circles. The proper tile size is defined according to the system bandwidth. The method significantly reduces the computational complexity of RT with no loss in accuracy. The method is verified by evaluating the power delay profile (PDP), delay spread and angular spread.
\end{abstract}

\section{Introduction}

Diffuse scattering has been shown to be a significant part of the propagation channel in wireless communications for both outdoor and indoor environments [1]. For statistical models, the residual power of the diffuse scattering components after subtracting the specular propagation paths can be estimated and modeled by the RiMAX algorithm $[2,3]$. For deterministic models, such as ray tracing (RT), the diffuse scattering components from the rough surfaces depends on the effective roughness[4,5], which has been validated with measurement data. It is known that the accuracy of RT comes at the cost of a high computational complexity, which directly scales with the number of propagation paths considered [6]. The diffuse scattering components constitute a high proportion of all the propagation paths, which is closely related to the diffuse scattering tile size. In [5], the size of each diffuse scattering tile is evaluated by recursively dividing the surface until the far-field condition is fulfilled. In [7], the surfaces are divided into 2272 diffuse scattering tiles within the size $0.5 \times 0.5 \mathrm{~m}^{2}$. It is obvious that the obtained diffuse scattering tiles of all these mentioned subdivision methods are related to a specific scenario. Moreover, the positions of the diffuse scattering tiles are fixed if the entire scenario, including the locations of the Tx and Rx and the objects, is not changed.

In this paper, we develop an effective subdivision algorithm for diffuse scattering of RT in indoor scenarios. The method can be used as a general procedure to subdivide rough surfaces. The main contributions are as follows:

- The algorithm consists of two main parts: i) the generation of diffuse scattering tiles, which fully demonstrates the random characteristic of diffuse scattering, and ii) the proper tile size calculation, which is related to the system bandwidth and is scenario independent.

- The accuracy of the proposed algorithm is tested by comparing with the proven subdivision algorithm of diffuse scattering theory [5]. The numeric simulations include the power delay profile (PDP), delay spread and angular spread.

- The computational complexity of diffuse scattering in RT can be reduced with no loss in accuracy.

\section{RT Channel Model}

The RT tool in the present work is a three-dimensional (3D) model and three major wave propagation mechanisms are taken into consideration: (i) line of sight (LOS), (ii) specular components as well as (iii) diffuse scattering. The specular components refer to reflection, penetration and diffraction contributions [6]. A directive pattern model is used to model the incoherent diffuse part of the channel in the present work, which assumes that the scattering lobe is steered towards the direction of the specular reflection [4]. The rough surface needs to be divided into multiple tiles for the diffuse scattering components calculation. The tile size is evaluated in [5] by recursively dividing the surface until the far-field condition is satisfied. In case the terminal is very close to the surface of an object, the algorithm of [5] would lead to a large amount of tiles within the infinitesimal sizes, which in turn results in the extremely high computational complexity. Moreover, the diffuse scattering tiles would be fixed if the entire environment is not changed, which can not express the random characteristic of the diffuse scattering components adequately. 


\section{New Subdivision Algorithm}

A new subdivision algorithm, based on concentric circles, is developed in the present work. It is assumed that the diffuse scattering tiles are the equally sized circular segments within the same radius $\Delta d$ on a certain surface and the the diffuse scattering path originates from the center of each tile.

\subsection{Generation of Diffuse Scattering Tiles}

The generation algorithm of the diffuse scattering tiles is shown in Fig. 1, whose procedure is illustrated as following:

Firstly, a random point $c_{\mathrm{S}}$ is placed on the rough surface as the center point of the concentric circles, whose coordinate is indicated as $\left(x_{\mathrm{c}}, y_{\mathrm{c}}, z_{\mathrm{c}}\right)$. The radius $r_{n}$ of these concentric circles are defined as

$$
r_{n}= \begin{cases}\Delta d & \text { if } n=0 \\ 2 n \cdot \Delta d & \text { if } n=1, \ldots, N\end{cases}
$$

where $n$ is the index of the concentric circle and $N$ is the number of concentric circles. The distance $r_{\max }$ between the center $c_{\mathrm{s}}$ and the furthest vertex of the rectangular surface can be obtained, which helps to determine

$$
N=\left\lfloor\frac{r_{\max }}{2 \cdot \Delta d}\right\rfloor
$$

where $\lfloor\cdot\rfloor$ takes the next smallest integer toward minus infinity.

Secondly, starting from $n=1$, circular scattering tiles are generated on each concentric circle separately. The first diffuse scattering tile at each concentric circle, whose center is denoted as $c_{\mathrm{n}, 0}$, is located at a random angle $\theta_{n, 0}$ within the region $[0,2 \pi)$. Based on the triangular relationship, the angle resolution $\theta_{n}$, which is the angle difference between two adjacent tile centers on the same concentric circle, can be calculated as

$$
\theta_{n}=2 \arcsin \left(\frac{1}{2 \cdot n}\right), n=1, \ldots, N
$$

Furthermore, the corresponding coordinate of the tile center $\left(x_{n, \mathrm{~s}}, y_{n, \mathrm{~s}}, z_{n, \mathrm{~s}}\right)$ can be obtained. For example, if the surface is horizontal, the coordinate is calculated as

$$
\left\{\begin{array}{l}
x_{n, \mathrm{~s}}=x_{\mathrm{c}}+r_{n} \cdot \cos \theta \\
y_{n, \mathrm{~s}}=y_{\mathrm{c}}+r_{n} \cdot \sin \theta \\
z_{n, \mathrm{~s}}=z_{\mathrm{c}}
\end{array}\right.
$$

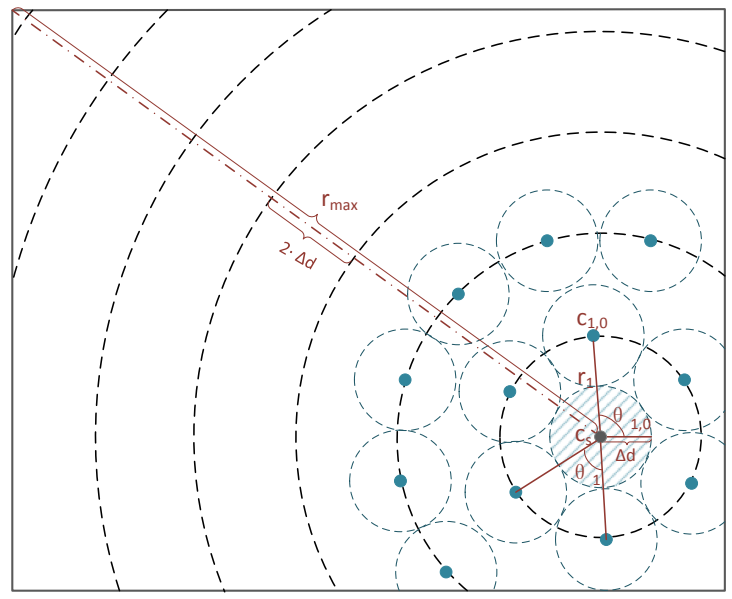

Figure 1: The principle of the generation of diffuse scattering tiles.

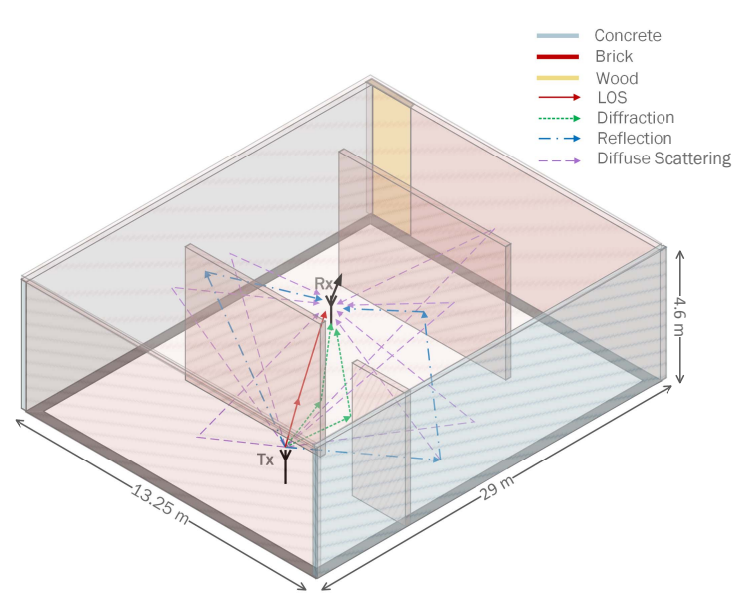

Figure 2: An indoor scenario for testing the performance of the proposed subdivision algorithm.

where $\theta=\theta_{n, 0}+l_{\mathrm{c}, n} \cdot \theta_{n}$ and $l_{\mathrm{c}, n} \in\left[1, N_{\mathrm{c}, n}\right]$ is the index of the circular tiles on each concentric circle, where $N_{\mathrm{c}, n}$ is the number of the circular tiles, defined as

$$
N_{\mathrm{c}, n}=\left\lfloor\frac{2 \pi}{\theta_{n}}\right\rfloor .
$$

The procedure described above is repeated until reaching the last concentric circle. The random angle $\theta_{n, 0}$ should be regenerated for each concentric circle. It should be noticed that some circular tiles are too close to the edge and the portions outside the surface are cut off. However, we still assume the size of those incomplete circular tiles as $d S$. The unused areas among the obtained circular tiles can make this compensation, which guarantees that the surface is not over occupied. 


\subsection{Proper Tile Size Calculation}

The remaining problem is how to determine a proper tile size $d S$, which is scenario independent. We propose an approach, which depends on the system bandwidth $B$. Strictly speaking, it should be the bandwidth used in the RT simulation ${ }^{1}$. The tile size is expressed as

$$
d S=\pi \cdot \Delta d^{2}=\pi \cdot\left(\frac{c}{2 \cdot B}\right)^{2} .
$$

It is known that the delay resolution is defined as $1 / B$. Therefore, this calculation means that the distance between any two circular tile centers on one surface is not shorter than the distance which is corresponding to the delay resolution. This condition serves to ensure that any diffuse scattering paths on one surface would not be overlapped in the same delay bin.

\section{Simulation Configuration}

In order to show the performance of the proposed subdivision algorithm, an indoor scenario which is shown in Fig. 2 is generated. The dimension of the room is about $29 \mathrm{~m} \times 13.25 \mathrm{~m} \times 4.6 \mathrm{~m}$. Simulation parameters can be found in [6]. We take into account single-bounce scattering, reflection-scattering and scattering-reflection cases in all simulations. Each diffuse scattering process is associated with a uniformly distributed random phase. In order to overcome the randomness, the channel simulation at each time sample is averaged over five realizations [1]. Therefore, there are 50 channel realizations obtained in total at the simulated Rx positions $N_{\mathrm{Rx}}=10$. According to the simulation parameters,

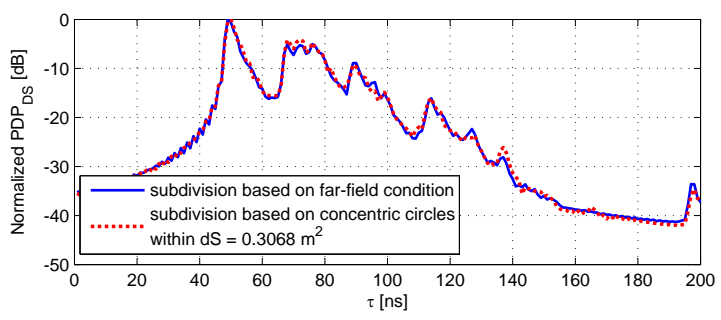

Figure 3: Normalized PDP $_{\mathrm{DS}}$ comparison between the subdivision based on the far-field condition and the concentric circles within $d S=0.3068 \mathrm{~m}^{2}$, respectively. $d S=0.3068 \mathrm{~m}^{2}$ for the proposed subdivision algorithm based on (6).

\section{Numerical Results}

PDP Comparison: Based on our numerical implementation, we compare the normalized PDP of the diffuse scattering paths $\mathrm{PDP}_{\mathrm{DS}}$ with different subdivision algorithms. The results are shown in Fig. 3, from which it can be observed that the $\mathrm{PDP}_{\mathrm{DS}}$ based on the concentric circles with $d S=0.3068 \mathrm{~m}^{2}$ is similar to the one based on the far-field condition. The correlation coefficient of these two different PDP $\mathrm{DS}_{\mathrm{DS}}$ is 0.986 .

Delay Spread Comparison: Delay spread $\sigma_{\tau}$ at one Rx's position, a measure of time dispersion of the channel, is defined as in [9]. The difference between the subdivision based on the far-field condition and the concentric circles is presented by the mean relative error (MRE)

$$
\operatorname{err}_{\tau}=\frac{1}{N_{\mathrm{Rx}}} \sum_{n_{\mathrm{Rx}}=1}^{N_{\mathrm{Rx}}} \frac{\left|\sigma_{\tau, \mathrm{far}}\left(n_{\mathrm{Rx}}\right)-\sigma_{\tau, \mathrm{cir}}\left(n_{\mathrm{Rx}}\right)\right|}{\sigma_{\tau, \mathrm{far}}\left(n_{\mathrm{Rx}}\right)},
$$

where $n_{\mathrm{Rx}}$ is the index of the Rx's position, $\sigma_{\tau, \text { far }}$ and $\sigma_{\tau, \text { cir }}$ are the delay spreads corresponding to the subdivision algorithm based on the far-filed condition and the concentric circles, respectively.

The delay spread $\sigma_{\tau}$ comparison according to different Rx's positions $n_{\mathrm{Rx}}$ between the subdivision based on

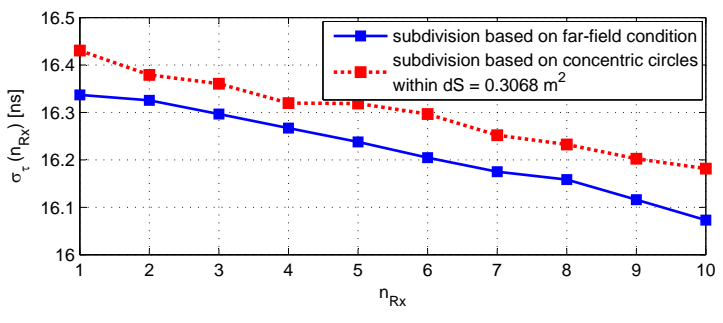

Figure 4: Delay spread $\sigma_{\tau}$ comparison according to different Rx's positions $n_{\mathrm{Rx}}$ between the subdivision based on the far-field condition and the concentric circles within $d S=0.3068 \mathrm{~m}^{2}$, respectively. the far-field condition and the concentric circles within $d S=0.3068 \mathrm{~m}^{2}$ is shown in Fig. 4. It can be seen that the delay spread difference between these two algorithms is always smaller than $0.1 \mathrm{~ns}$. Moreover, the $\operatorname{err}_{\tau}$ is about $-25 \mathrm{~dB}$ between these two subdivision algorithms.

Angular Spread Comparison: For the angular spread comparison, the method is defined similarly as the delay

\footnotetext{
${ }^{1}$ The bandwidth of the sub-band $B_{\text {sub }}$ is applied in the sub-band divided RT for an ultra-wideband (UWB) case [8].
} 
spread comparison. The angular spread analysis includes i) the azimuth of arrival (AoA) spread and ii) the elevation of arrival (EoA) spread. Fig. 5 (a) gives the AoA spread $\sigma_{\mathrm{AoA}, \phi}$ comparison result according to different Rx's positions $n_{\mathrm{Rx}}$ between the subdivision based on the far-field condition and the concentric circles within $d S=0.3068 \mathrm{~m}^{2}$. The AoA spread variation based on two subdivision algorithms is always smaller than $0.2^{\circ}$. The EoA spread $\sigma_{\mathrm{EoA}, \phi}$ comparison result is given in Fig. 5 (b). The $\sigma_{\mathrm{EoA}, \phi}$ variation is always smaller than $0.05^{\circ}$. Furthermore, the $\operatorname{err}_{\mathrm{AoA}, \phi}$ and $\operatorname{err}_{\mathrm{EoA}, \phi}$ are about $-35 \mathrm{~dB}$ and $-27.5 \mathrm{~dB}$ when $d S=0.3068 \mathrm{~m}^{2}$, respectively.

Computational Complexity: The numerical complexity is significantly reduced within the proposed subdivision algorithm. Considering one Rx's position, there are 37889 and 21026 diffuse scattering paths obtained by the subdivision algorithms based on the far-field condition and the concentric circles within $d S=0.3068 \mathrm{~m}^{2}$, respectively. The corresponding simulation time is $81 \mathrm{~s}$ and $41 \mathrm{~s}$ (2.4GHz Intel Core i5 CPU with 4GB RAM). Compared with the subdivision algorithm based on the far-field condition, it can be analyzed that about $45 \%$ simulation time is saved based on the proposed subdivision algorithm.

\section{Conclusion}

In this paper, we presented an effective subdivision algorithm for diffuse scattering of RT in indoor scenario. The algorithm is generated based on the concentric circles and the system bandwidth. The random characteristic of the diffuse scattering is demonstrated and the tile size is independent of the scenario. The new algorithm based on the concentric circles is verified by comparing its results with the one based on the far-field conditions through evaluating: (i) the normalized PDPs of both methods shows a correlation of 0.968 , (ii) the MRE of the delay spread is about $-25 \mathrm{~dB}$ between these two subdivision algorithms and (iii) the MREs of the AoA and the EoA spread are about $-35 \mathrm{~dB}$ and $-27.5 \mathrm{~dB}$ between the mentioned two subdivision algorithms, respectively. Furthermore, the computational complexity is significantly reduced because of the smaller number of the diffuse scattering paths.

\section{References}

[1] F. Mani, F. Quitin, and C. Oestges, "Directional spreads of dense multipath components in indoor environments: Experimental validation of a ray-tracing approach," Antennas and Propagation, IEEE Transactions on, vol. 60, no. 7, pp. 3389 - 3396, July 2012.

[2] A. Richter, "RiMAX - a flexible algorithm for channel parameter estimation from channel sounding measurements," in temporary document TD(04)045 presented at COST 273 Meeting, Jan. 2004.

[3] D. Gaillot, E. Tanghe, P. Stefanut, W. Joseph, M. Lienard, P. Degauque, and L. Martens, "Accuracy of specular path estimates with ESPRIT and RiMAX in the presence of measurement-based diffuse multipath components," in Antennas and Propagation (EUCAP), Proceedings of the 5th European Conference on, April 2011, pp. 3619-3622.

[4] V. Degli-Esposti, F. Fuschini, E. M. Vitucci, and G. Falciasecca, "Measurement and modelling of scattering from buildings," Antennas and Propagation, IEEE Transactions on, vol. 55, no. 1, pp. 143 - 153, Jan. 2007.

[5] F. Mani, F. Quitin, and C. Oestges, "Accuracy of depolarization and delay spread predictions using advanced ray-based modeling in indoor scenarios," EURASIP Jounal in Wireless Commmunications and Networking, vol. 2011, p. 11, 2011.

[6] M. Gan, F. Mani, F. Kaltenberger, C. Oestges, and T. Zemen, "A ray tracing algorithm using the discrete prolate spheroidal subspace," in IEEE International Conference on Communications (ICC), June 2013.

[7] O. Franek, J. Andersen, and G. Pedersen, "Diffuse scattering model of indoor wideband propagation," Antennas and Propagation, IEEE Transactions on, vol. 59, no. 8, pp. 3006-3012, Aug. 2011.

[8] M. Gan, P. Meissner, F. Mani, E. Leitinger, M. Froehle, C. Oestges, K. Witrisal, and T. Zemen, "Low-complexity sub-band divided ray tracing for UWB indoor channel," in IEEE Wireless Communications and Networking Conference (WCNC), April 2014 , to be presented.

[9] P. Petrus, J. Reed, and T. Rappaport, "Geometrical-based statistical macrocell channel model for mobile environments," Communications, IEEE Transactions on, vol. 50, no. 3, pp. 495-502, Mar. 2002. 